Sonderdruck aus

\title{
Archiv der Pharmazie
}

316. Band, Heft 9, Seite $737-746$

Verlag Chemie GmbH, Weinheim (Bergstr.)

Boran-Addukte des Narkotins und Hydrastins und ihrer Reduktionsprodukte

Silvia Prior und Wolfgang Wiegrebe* 


\title{
Archiv der Pharmazie
}

316. Band

September 1983

Heft 9

Arch. Pharm. (Weinheim) 316, 737-746 (1983)

\section{Boran-Addukte des Narkotins und Hydrastins und ihrer Reduktionsprodukte}

\author{
Silvia Prior und Wolfgang Wiegrebe* \\ Institut für Pharmazie der Universität Regensburg, Postfach 397, D-8400 Regensburg \\ Eingegangen am 15. Juli 1982
}

$\alpha$-Narkotin (3), $\beta$-Hydrastin (6) und ihre Reduktionsprodukte binden Boran koordinativ am Stickstoff. - Aus $\alpha$-Narkotindiol (8) entsteht mit K-tert. butylat/Benzophenon nicht $\alpha$-Anhydronarkotindiol $^{7}$, sondern eine Quettamin-ähnliche Verbindung 9 ohne Racemisierung am Benzyl-C.

\section{Borane Adducts of Narcotine, Hydrastine and Their Reduction Products}

$\alpha$-Narcotine (3), $\beta$-Hydrastine (6) and their reduction products bind $\mathrm{BH}_{3}$ coordinatively at the $\mathrm{N}$ atom. $\alpha$-Narcotinediol (8) when treated with potassium tert.butylate and benzophenone does not yield $\alpha$-anhydronarcotinediol ${ }^{7)}$ but leads to a quettamine-like compound 9 without racemisation at the benzyl $\mathrm{C}$ atom.

Im Zusammenhang mit unseren Arbeiten zum Mechanismus der Umsetzung von 1-(o-Hydroxymethylbenzyl)-N-methyl-1,2,3,4-tetrahydroisochinolinen (Typ 1) zu 3-Phenylisochromanen (Typ 2) ${ }^{1)}$ setzten wir $\alpha$-Narkotin (3) mit verschiedenen Boran-Derivaten um.

Aus 3 entstand mit überschüssigem Disiamylboran ${ }^{2)}$ ein Produkt, das eine $\gamma$-Lactonbande bei $1750 \mathrm{~cm}^{-1}$ und charakteristische Banden zwischen 2300 und $2400 \mathrm{~cm}^{-13)}$ aufweist. Das ${ }^{1} \mathrm{H}$-NMR-Spektrum zeigt alle Signale des $\alpha$-Narkotins (3) (s. Tab. 1), doch ist eine $\mathrm{OCH}_{3}$-Gruppe stark nach höherem Feld verschoben $(\delta=3.20 \mathrm{ppm})$. Auffallend sind weiterhin die geringe Aufspaltung des AB-Signals der beiden Aromaten-H am Benzylrest und die fehlende Kopplung der benzylischen Protonen $(\delta=4.55$ und $\delta=6.88 \mathrm{ppm})$, von denen das H-9-Signal stark nach tieferem Feld verschoben ist. Die nach den IR-Banden zwischen 2300 und $2400 \mathrm{~cm}^{-1}$ wahrscheinliche Struktur eines $\alpha$-Narkotin-Boranadduktes $\left(3-\mathrm{BH}_{3}\right)$ wird ms gestützt: Felddesorption (FD) zeigt $\mathrm{M}^{+\bullet}$ bei $\mathrm{m} / \mathrm{z} 427\left(3-\mathrm{BH}_{3}\right)$ und $\mathrm{m} / \mathrm{z} 425$ $\left(3-\mathrm{BH}^{+\bullet}\right)$. Im MS $(70 \mathrm{eV})$ tritt als Basepeak das Isochinolinbruchstück bei $\mathrm{m} / \mathrm{z} 220$ auf, das $\mathrm{CH}_{3}{ }^{\bullet} \mathrm{zu} \mathrm{m} / \mathrm{z} 205$ abspaltet. $-3-\mathrm{BH}_{3}$ läßt sich mit 20 proz. $\mathrm{KOH}$ zu 3 spalten.

3 wurde daraufhin mit $\mathrm{NaBH}_{4}$ und überschüssigem $\mathrm{BF}_{3}$-Etherat ${ }^{4)}$ reduziert; eines der beiden entstandenen Produkte zeigt keine Lacton- und Hydroxylbanden, aber Boranbanden zwischen 2300 und $2400 \mathrm{~cm}^{-13}$ ). Im ${ }^{1} \mathrm{H}$-NMR-Spektrum (Abb. 2) ist die nach höherem 

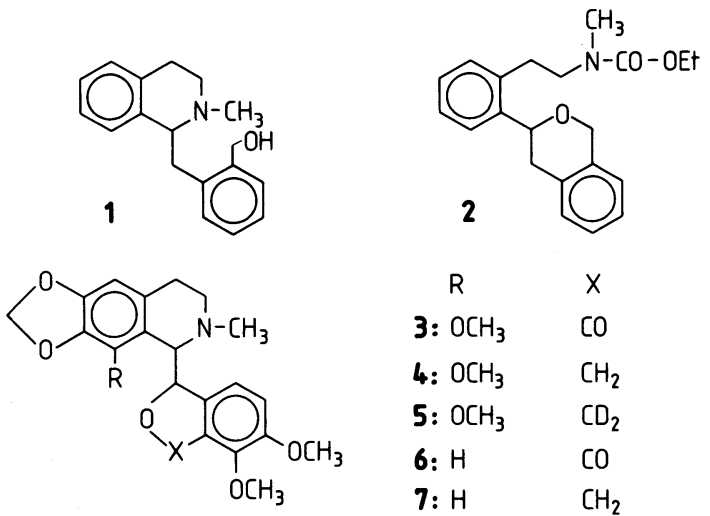

3: $\mathrm{OCH}_{3} \quad \mathrm{CO}$

4: $\mathrm{OCH}_{3} \quad \mathrm{CH}_{2}$

5: $\mathrm{OCH}_{3} \quad \mathrm{CD}_{2}$

6: $\mathrm{H}$

$\mathrm{CO}$

7: $\mathrm{H} \quad \mathrm{CH}_{2}$

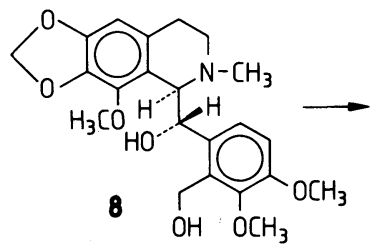

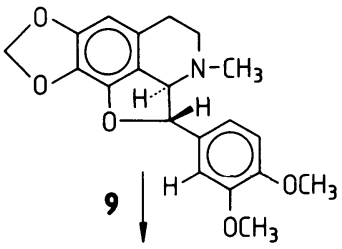<smiles>COc1ccc2c(c1O)C(Cc1ccc(O)cc1)N(C)CC2</smiles><smiles></smiles>

Abb. 1

Feld verschobene $\mathrm{OCH}_{3}$-Gruppe zu erkennen; $\mathrm{H}-1$ und $\mathrm{H}-1$ ' treten als Singuletts mit fast gleicher chem. Verschiebung wie im $3-\mathrm{BH}_{3}$-Addukt auf. Charakteristisch ist ein AB-System der zwei C-3-Protonen, dessen einer Teil zu einem Doppeldublett aufspaltet. Danach handelt es sich um das $\alpha$-Anhydronarkotindiol-Boranaddukt $\left(4-\mathrm{BH}_{3}\right)$. Durch FD findet man $\mathrm{M}^{+\bullet}$ bei $\mathrm{m} / \mathrm{z} 413\left(4-\mathrm{BH}_{3}\right)$ und $\mathrm{m} / \mathrm{z} 411\left(4-\mathrm{BH}^{+\bullet}\right)$. Das EI-MS zeigt als Basepeak $\mathrm{m} / \mathrm{z} 220$.

Beim Umkristallisieren bzw. durch Erhitzen mit 20 proz. $\mathrm{KOH}$ wird 4-BH $\mathrm{Bu}_{3} \mathrm{zu}$ gespalten; sein ${ }^{1} \mathrm{H}$-NMR-Spektrum (Abb. 3) zeigt ein Dublett für die Protonen an C-3 $(\delta=$ $\left.5.16 \mathrm{ppm}, \mathrm{J}_{\mathrm{gem}}=2 \mathrm{~Hz}\right)$ und die Dubletts für $\mathrm{H}-1$ und $\mathrm{H}-1^{\prime}(\mathrm{J}=4 \mathrm{~Hz}$ bei $\delta=5.38$ und $4.26 \mathrm{ppm}$ ). Durch FD wurde $\mathrm{M}^{+\bullet}$ bei $\mathrm{m} / \mathrm{z} 399$ gefunden. Die spektroskopischen Daten entsprechen dem freien 1-Tetrahydroisochinolyl-isobenzofuran 4. Pettit et al. ${ }^{4)}$ haben $\gamma$-Lactone in Steroiden mit $\mathrm{LiALH}_{4} / \mathrm{BF}_{3}$-Etherat bzw. $\mathrm{NaBH}_{4} / \mathrm{BF}_{3}$-Etherat zu entspr. Tetrahydrofuranen reduziert. Das zweite Produkt war mit 3- $\mathrm{BH}_{3}$ aus der Umsetzung von 3 mit Disiamylboran (s.o.) identisch. Die Umsetzung von 3 mit LiAlH$/{ }_{4} / \mathrm{BF}_{3}$-Etherat führt zu den gleichen Produkten. Der Versuch, 3 mit Diboran zu reduzieren, lieferte ebenfalls 3- $\mathrm{BH}_{3}$, das nicht weiter reagiert.

Um die Signale des ${ }^{1} \mathrm{H}-\mathrm{NMR}$-Spektrums von $4-\mathrm{BH}_{3}$ eindeutig zuzuordnen, wurde 3 mit deuterierten Hydriden gleichermaßen umgesetzt. Aus 3 entstand mit $\mathrm{NaBD}_{4} / \mathrm{BF}_{3}$-Etherat 
Tab. 1: ${ }^{1} H-N M R$-Spektren $\left(\mathrm{CDCl}_{3}\right)$

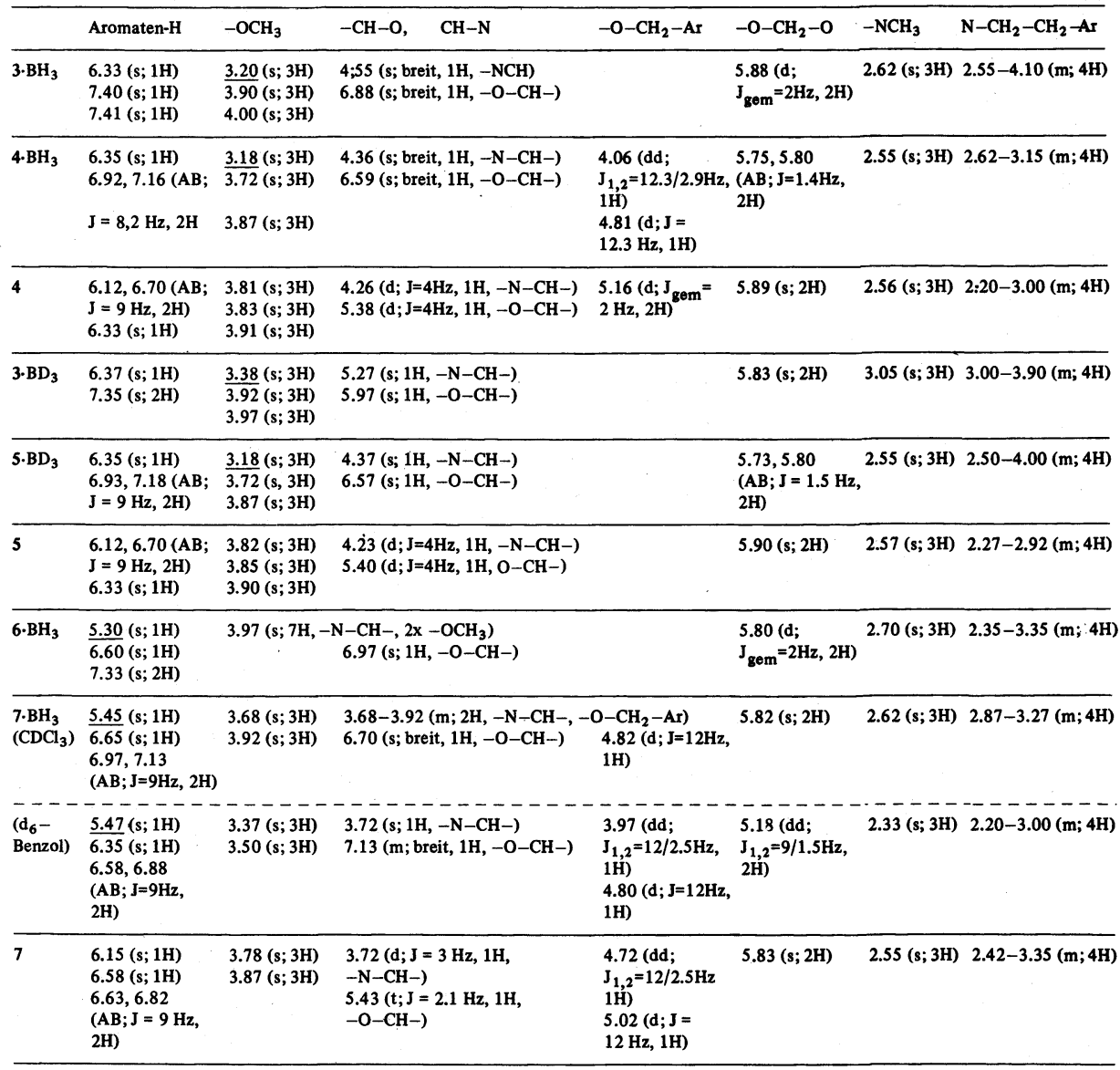

ein Produkt, dessen ${ }^{1} \mathrm{H}-\mathrm{NMR}$-Spektrum dem des $3-\mathrm{BH}_{3}$ sehr ähnlich ist, und das im IR-Spektrum eine $\gamma$-Lactonbande aufweist. Da keine Boranbande vorliegt, konnte es sich nur um ein Bortrideuterid- oder Bortrifluorid-Addukt des $\alpha$-Narkotins (3) handeln: Durch FD wurde $\mathrm{m} / \mathrm{z} 430\left(3-\mathrm{BD}_{3}\right)$ gefunden.

3 setzt sich mit LiAlD $4 / \mathrm{BF}_{3}$-Etherat zu einer Verbindung um, die als Bortrideuterid-Addukt vorliegt, da das ${ }^{1} \mathrm{H}-\mathrm{NMR}$-Spektrum eine deutlich nach höherem Feld verschobene

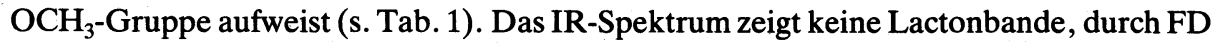
findet $\operatorname{man} \mathrm{M}^{+\bullet}$ bei $\mathrm{m} / \mathrm{z}$ 418: das Produkt ist somit ein dideuteriertes 4-Bortrideuterid-Addukt $\left(5-\mathrm{BD}_{3}\right)$. Es wird durch $\mathrm{KOH}$ zu 5 gespalten, das durch $\mathrm{FD}(\mathrm{m} / \mathrm{z} 401)$ und sein ${ }^{1} \mathrm{H}$-NMR-Spektrum (s. Tab. 1) identifiziert wurde. Ein Vergleich der ${ }^{1} \mathrm{H}-\mathrm{NMR}$-Spektren von $3-\mathrm{BH}_{3}, 5-\mathrm{BD}_{3}, 4$ und 5 (s. Tab. 1) zeigt, daß das nochmals aufgespaltene AB-System von 4- $\mathrm{BH}_{3}$ der $\mathrm{CH}_{2}$-Gruppe des Isobenzofuranrings zuzuordnen ist. Diese Methylengruppe, die in 4 als Dublett bei $\delta=5.16$ ppm auftritt, fehlt in 5 . 
<smiles>COc1ccc2c(c1OC)COC2[C+]1NCCc2cc3c(c(OC)c21)OCO3</smiles>

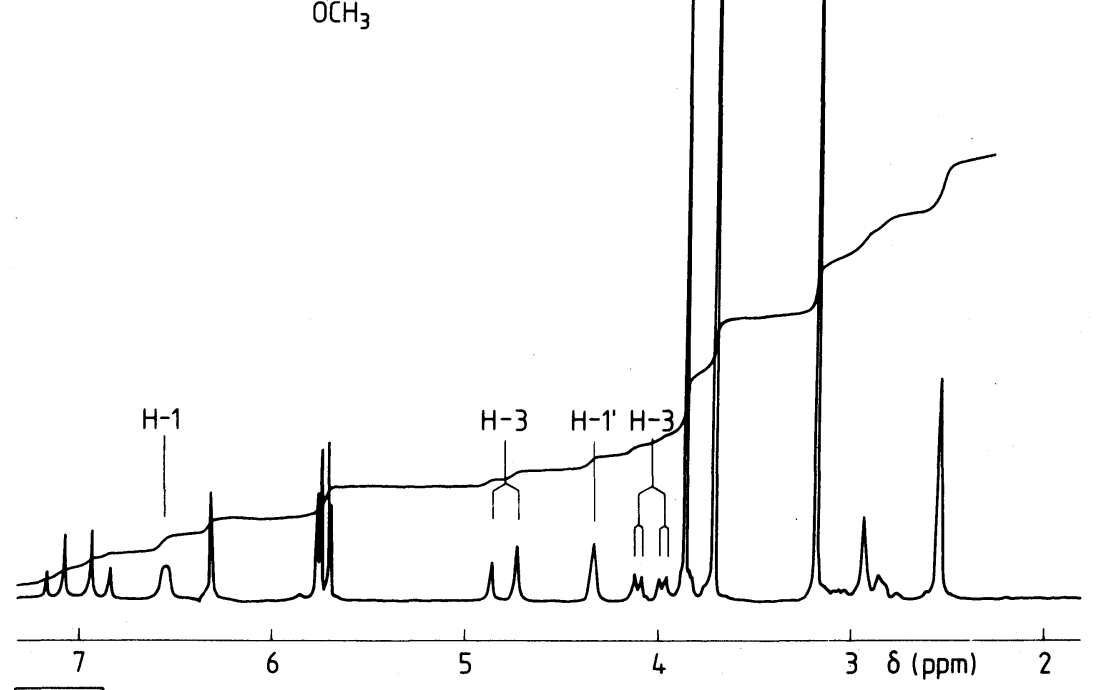

Ph644.2

Abb. 2: $90 \mathrm{MHz}-S p e k t r u m$ von 4-BH

Die Umsetzung von $\alpha$-Narkotin (3) mit Boranen führt also entweder zu 3-Boran-1:1-Addukten oder zu den entspr. Isobenzofuranen bzw. deren Boranaddukten.

Offenbar ist die 8-Methoxygruppe des $\alpha$-Narkotins (3) nicht an der Boran-Addukt-Bildung beteiligt: Aus $\beta$-Hydrastin (6) entstanden mit $\mathrm{NaBH}_{4} / \mathrm{BF}_{3}$-Etherat durch Variation der Reaktionsbedingungen die zu $\alpha$-Narkotin (3) analogen Produkte: $\beta$-Hydrastin-Boranaddukt $\left(6-\mathrm{BH}_{3}\right), \beta$-Anhydrohydrastindiol-Boranaddukt $\left(7-\mathrm{BH}_{3}\right)$ und freies $\beta$-Anhydrohydrastindiol (7), deren Strukturen durch ihre spektroskopischen Daten gesichert wurden. Die chemischen Verschiebungen der Methoxygruppen im Benzylteil von 6- $\mathrm{BH}_{3}$ und 7- $\mathrm{BH}_{3}$ bleiben gegenüber $\beta$-Hydrastin (6) und freiem 7 nahezu gleich. Auffallend ist dagegen die Hochfeldverschiebung von $\mathrm{H}-8$ in $6-\mathrm{BH}_{3}$ und 7- $\mathrm{BH}_{3}$ (s. Tab. 1).

$\beta$-Hydrastin (6) liegt in Konformation A (Abb. 4) vor ${ }^{5}$, während $\alpha$-Narkotin (3) durch die Methoxygruppe in 8-Stellung die Konformation B (Abb. 4) einnimmt ${ }^{6}$. Durch die Adduktbildung mit $\mathrm{BH}_{3}$ wird es in Konformation $\mathbf{A}$ gezwungen -das erklärt die Verschiebung der $\mathrm{C}-8-\mathrm{OCH}_{3}$-Gruppe zu höherem Feld durch den abschirmenden Effekt des Aromaten. Im $\beta$-Hydrastin-Boranaddukt $\left(6-\mathrm{BH}_{3}\right)$ und im Isobenzofuran-Boranaddukt 7- $\mathrm{BH}_{3}$ erklärt die stärkere Abschirmung die Verschiebung von $\mathrm{H}-8$ nach höherem Feld. Wir nehmen deshalb an, daß das Boran über den Stickstoff koordinativ gebunden wird (vgl. ${ }^{3)}$ ). 


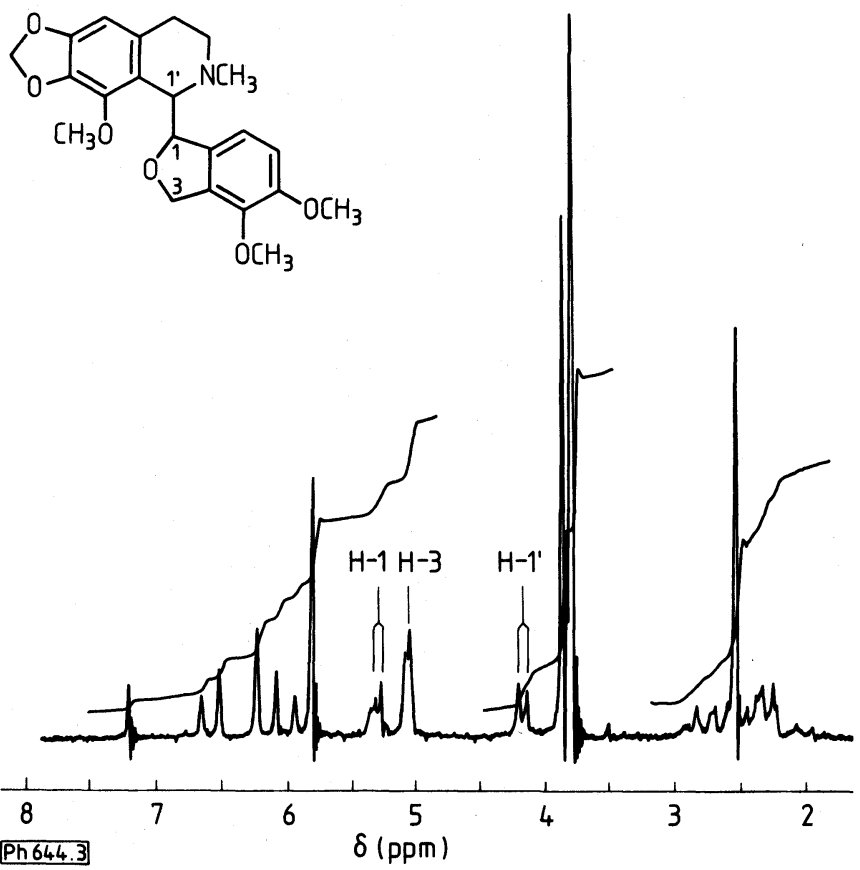

Abb. 3: $60 \mathrm{MHz}$-Spektrum von 4

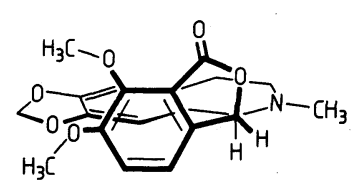

Ph644.4 A

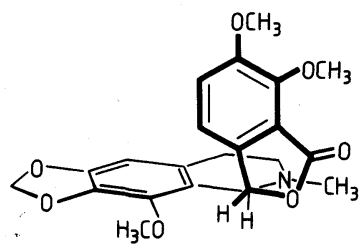

B
Abb. 4:

Bentley $^{7)}$ erhielt bei der Umsetzung von $\alpha$-Narkotindiol (8) mit Benzophenon und K-tert. butylat eine Verbindung, die er als $\alpha$-Anhydronarkotindiol $\left(, 4^{\text {“" }}\right)$, bezeichnete, deren Daten aber nicht mit 4 übereinstimmen. Beim Nacharbeiten erhielten wir neben 3 eine Substanz, die nach Schmp. und optischer Drehung weder mit Bentley's Anhydronarkotindiol, noch mit 4 identisch ist. Ihr ${ }^{1} \mathrm{H}-\mathrm{NMR}$-Spektrum (Abb.5) zeigt die Methylendioxy-Gruppe als AB-System. Gut erkennbar sind zwei Dubletts der tert. benzylischen Protonen, von denen ein Dublett nochmals zu zwei Tripletts aufgrund einer long-range-Kopplung aufspaltet. Ein drittes aromatisches Proton im Benzylteil des Moleküls (m-Kopplung erkennbar) deutet auf den Verlust der Hydroxymethylseitenkette hin. Das Fehlen einer Methoxygruppe legt die Spaltung einer Phenolethergruppierung nahe, da aber im IR-Spektrum keine phenolische OH-Bande erkennbar ist, und die Substanz nicht als Phenol reagiert, muß der Phenol-Sauerstoff mit dem $\alpha$-C-Atom 


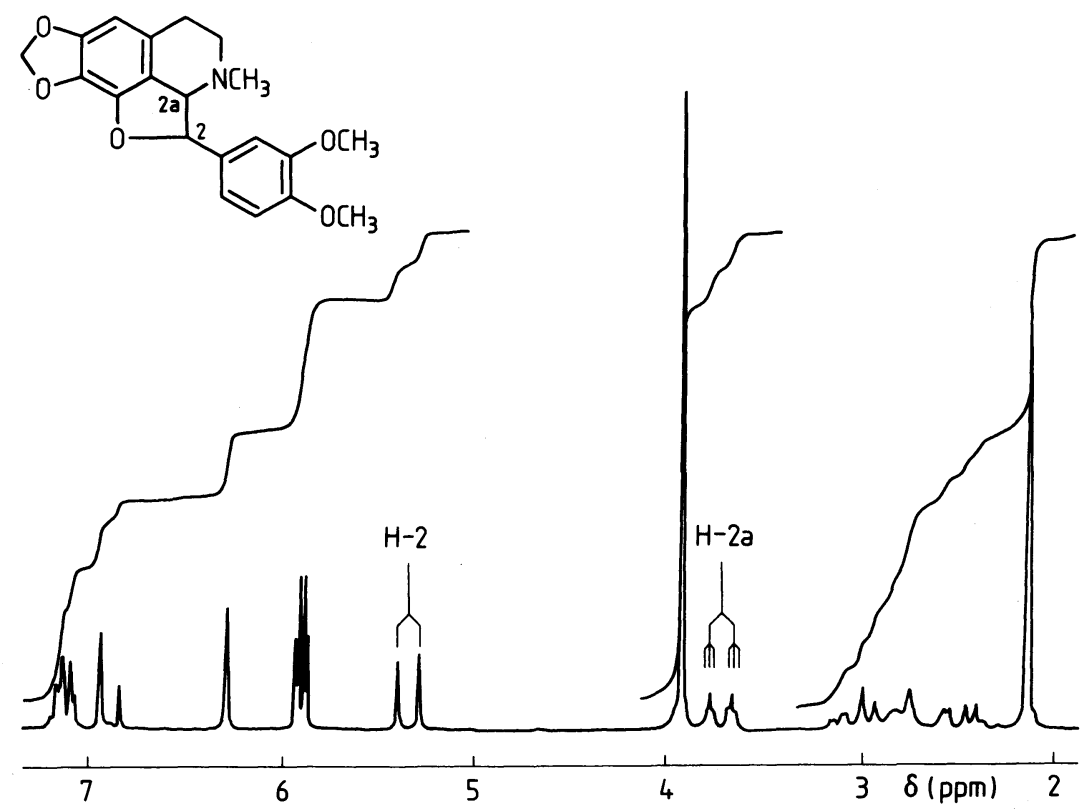

Ph644.5

Abb. $590 \mathrm{MHz}$-Spektrum von 9

verknüpft worden sein: Alle Daten treffen auf Struktur 9 zu. - MS und Hochauflösung (HR) von $\mathrm{M}^{+\bullet}$ bei m/z 355 (Abb. 6) stützen diese Annahme. Durch HR der Bruchstücke wird der Zerfall von $\mathrm{M}^{+\bullet}$ geklärt und die Struktur des Moleküls im Sinne von 9 bestätigt: $\mathrm{M}^{+\bullet}$ zerfällt (Abb. 6) unter $\mathrm{C}_{6} \mathrm{H}_{3}\left(\mathrm{OCH}_{3}\right)_{2}$-Abspaltung $\mathrm{zu} \mathrm{m} / \mathrm{z} 218$ und unter Verlust von Dimethoxybenzaldehyd zum Fragment m/z 189, das HCHO zu m/z 159 verliert.

Diese Reaktion bietet möglicherweise einen Zugang zu der von Shamma et al. ${ }^{899)}$ beschriebenen Alkaloidklasse der Quettamine, die biogenetisch aus Oblongin (10)-ähnlichen Alkaloiden durch Oxidation an C- $\alpha$ mit nachfolgendem Angriff des phenolischen C-8-OH am hydroxylierten C- $\alpha$ über ein Benzylcarbenium--Ion ${ }^{9)}$ unter Bildung des Dihydrofuran-Systems entstehen sollen ${ }^{8)}$. - Für die Entstehung des Dihydrobenzofurans 9 haben wir noch keine schlüssige Erklärung. Aufgrund des ${ }^{1} \mathrm{H}-\mathrm{NMR}$-Spektrums, das ein Enantiomer zeigt, verläuft die Reaktion nicht unter Racemisierung an C-2. Da die Kopplungskonstante für $\mathrm{H}-2$ und $\mathrm{H}-2 \mathrm{a}$ in $910.1 \mathrm{~Hz}$ beträgt, sind diese Protonen transständig.

9 wurde mit Methyliodid in 9- $\mathrm{CH}_{3} \mathrm{I}$ überführt, dessen Hofmann-Abbau das Styrol 11 gab. Beim Emde-Abbau von 9- $\mathrm{CH}_{3}$ konnte kein Produkt isoliert werden. Das Styrol 11 zeigt für H-2 und H-3 eine Kopplung von $2.5 \mathrm{~Hz}$, was die Annahme der trans-Anordnung in 9 bestätigt. Nach Modellbetrachtungen ist die gegenüber 9 veränderte Kopplungskonstante auf eine Verminderung der Ringspannung im 5-Ring mit entsprechenden Veränderungen des Winkels zwischen $\mathrm{H}-2$ und $\mathrm{H}-2 \mathrm{a}$ zu erklären (vgl. $\left.{ }^{10}\right)$. Aufgrund dieser Daten steht fest, daß die Reaktion von 8 zu 9 unter Retention abläuft. 
<smiles>CN1CCc2cc3c(cc2C1)OCO3</smiles>

$\mathrm{m} / \mathrm{z} 189(100 \%)$

$\mathrm{C}_{11} \mathrm{H}_{11} \mathrm{NO}_{2}$<smiles>CC(C)C=O</smiles>

$\mathrm{m} / \mathrm{z} 159(43 \%)$

$\mathrm{C}_{10} \mathrm{H}_{8} \mathrm{NO}$

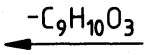<smiles>COc1ccc(C2Oc3c4c(cc5c3C2N(C)CC5)OCO4)cc1OC</smiles>

$9 \mathrm{~m} / \mathrm{z} 355(48 \%)$

$\mathrm{C}_{20} \mathrm{H}_{21} \mathrm{NO}_{5}$

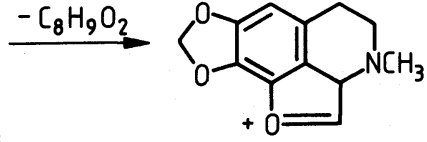

$\mathrm{m} / \mathrm{z} 218(46 \%)$

$\mathrm{C}_{12} \mathrm{H}_{12} \mathrm{NO}_{3}$

Abb. 6:

\section{Experimenteller Teil}

Allgemeine Angaben: Schmp.: Apparatur nach Tottoli (Büchi), (nicht korr.). Elementaranalysen: Mikroanalytisches Laboratorium der Universität Regensburg (Leiter: G. Wandinger). - IR: Beckman Acculab III. ${ }^{1} H$-NMR: Varian EM 360 A (60 MHz), Bruker WH 90 (90 MHz) und Varian EM 390; $\mathrm{CDCl}_{3}$, TMS inn. Stand. bei $30^{\circ}$. MS: Varian MAT CH 5; Anregungsenergie $70 \mathrm{eV}$. Signale mit einer rel. Intensität $<1 \%$ sind nur ausnahmsweise aufgeführt. Rel. Intensitäten und metastabile Ionen (*) sind in Klammern angegeben. Hochauflösende MS und FD: Varian MAT 311A. UV: Shimadzu 210, Uvikon 810 (Kontron), in Methanol (Uvasol ${ }^{\circledR}$ Merck) bei $10 \mathrm{~mm}$ Schichtdicke. Drehwerte: Polarimeter Perkin Elmer $241 \mathrm{MC}$; Schichtdicke $10 \mathrm{~cm}, \mathrm{c}=0,5$.

\section{$\alpha-N a r k o t i n-B o r a n a d d u k t\left(3-\mathrm{BH}_{3}\right)$}

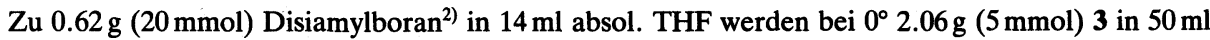
absol. THF getropft. Das Reaktionsgemisch wird $8 \mathrm{~d}$ bei $0^{\circ}$ gerührt, anschließend auf Eiswasser gegossen und mit $\mathrm{CHCl}_{3}$ extrahiert. Das nach Trocknen der Lösung erhaltene farblose Öl wird sc (Kieselgel; Essigester) getrennt. Fraktion Rf 0.7: farblose Kristalle von 3-BH . Ausb. $0.21 \mathrm{~g} \mathrm{(10 \% ),}$ Schmp. $155-157^{\circ},[\alpha]_{D}^{20}=+130^{\circ}\left(\mathrm{CHCl}_{3}\right) \cdot \mathrm{C}_{22} \mathrm{H}_{23} \mathrm{NO}_{7} \cdot \mathrm{BH}_{3}$ (427.3) Ber. C 61.8 H 6.13, Gef. C 61.4 H 6.03. IR (KBr): $1750(\mathrm{CO}), 2300-2400 \mathrm{~cm}^{-1}\left(\mathrm{BH}_{3}\right)$. MS: $\mathrm{m} / \mathrm{z}=235(8 \%), 220(100 \%), 205(12 \%$, $\left.{ }^{*} 191.02\right), 177\left(17 \%,{ }^{*} 152.82\right)$. MS-FD: $\mathrm{m} / \mathrm{z}=427\left(\mathrm{M}^{+\bullet}\right), 425$. UV (Methanol): $\lambda \max (\log \varepsilon)=222$ (4.24), 288 (sh; 3.49), $312 \mathrm{~nm}$ (3.51). Fraktion Rf 0.5: farblose Kristalle von 3 (1.70 g, $82 \%)$.

\section{3- $\mathrm{BH}_{3}, 1,3-D i h y d r o-4,5-d i m e t h o x y-1-(1,2,3,4-t e t r a h y d r o-8-m e t h o x y-2-m e t h y l-6,7-m e t h y l e n d i o x y-$} isochinolyl-1)-isobenzofuran ( $\alpha$-Anhydronarkotindiol) (4) und 4-BH

$0.83 \mathrm{~g}(2 \mathrm{mmol}) 3$ werden in $10 \mathrm{ml} \mathrm{BF}_{3}$-Etherat gelöst, langsam bei $0^{\circ} \mathrm{zu} 0.15 \mathrm{~g}(2 \mathrm{mmol}) \mathrm{NaBH}_{4}$ in $4 \mathrm{ml}$ absol. THF getropft und $1 \mathrm{~h}$ bei $0^{\circ}$ gerührt. Nach 2-stdg. Erhitzen unter Rückfluß und Abkühlen wird die Lösung auf Eiswasser gegossen; die Produkte werden mit $\mathrm{CHCl}_{3}$ extrahiert. Die Lösung wird mit $\mathrm{Na}_{2} \mathrm{SO}_{4}$ getrocknet und eingeengt, das Öl sc (Kieselgel; Essigester) getrennt.

4- $\mathrm{BH}_{3}$ : Fraktion $\mathrm{Rf} 0.8$ : farblose Kristalle aus Ether, Ausb. $0.53 \mathrm{~g}(64 \%)$, Schmp. $151^{\circ},[\alpha]_{\mathrm{D}}^{20}=$ $+114^{\circ}\left(\mathrm{CHCl}_{3}\right) \cdot \mathrm{C}_{22} \mathrm{H}_{25} \mathrm{NO}_{6} \cdot \mathrm{BH}_{3}$ (413.3) Ber. C $63.9 \mathrm{H} \mathrm{6.83,} \mathrm{Gef.} \mathrm{C} 63.5 \mathrm{H} 6.65$. IR (KBr): $2300-2400 \mathrm{~cm}^{-1}\left(\mathrm{BH}_{3}\right)$. MS: $\mathrm{m} / \mathrm{z}=412(22 \%), 398(2 \%), 396(4 \%), 384\left(12 \%,{ }^{*} 357.90\right), 382(6 \%)$, $282(5 \%), 233(27 \%), 232(42 \%), 220(100 \%), 205\left(73 \%,{ }^{*} 191.02\right), 179(73 \%)$. MS-FD: m/z = 413 $\left(\mathrm{M}^{+\bullet}\right), 411$. UV (Methanol): $\lambda \max (\log \varepsilon)=226(4.19), 280 \mathrm{~nm}(3.47)$. 


\section{4: Freisetzung von 4 aus 4- $\mathrm{BH}_{3}$ :}

a) Durch mehrmaliges Umkristallisieren aus EtOH erhält man fast quantitativ 4.

b) $0.59 \mathrm{~g}(1.2 \mathrm{mmol})$ 4-BH 3 werden in $20 \mathrm{ml} 20$-proz. $\mathrm{KOH} 2 \mathrm{~h}$ unter Rückfluß erhitzt. Nach Abkühlen und Neutralisieren wird das Produkt mit $\mathrm{CHCl}_{3}$ extrahiert, die Lösung mit $\mathrm{Na}_{2} \mathrm{SO}_{4}$ getrocknet und eingeengt. Aus Ether kristallisiert 4, das aus Ethanol umkristallisiert wird. Ausb. $0.43 \mathrm{~g}(90 \%)$, Schmp. $126^{\circ},[\alpha]_{\mathrm{D}}^{20}=-112^{\circ}\left(\mathrm{CHCl}_{3}\right) . \mathrm{C}_{22} \mathrm{H}_{25} \mathrm{NO}_{6}(399.4)$ Ber. C $66.2 \mathrm{H} 6.31 \mathrm{~N} 3.5$, Gef. C 65.6 H 6.21 N 3.6. MS: $\mathrm{m} / \mathrm{z}=220(100 \%), 205(18 \%), 179(1 \%)$. MS-FD: $\mathrm{m} / \mathrm{z}=399\left(\mathrm{M}^{+\bullet}\right)$. UV (Methanol): $\lambda \max (\log \varepsilon)=218(4.36), 280 \mathrm{~nm}(3.47)$.

Fraktion Rf 0.7: farblose Kristalle von $3-\mathrm{BH}_{3}(0.05 \mathrm{~g}, 6 \%)$.

\section{3- $\mathrm{BH}_{3}$ aus 3 und Diboran}

$0.41 \mathrm{~g}(1 \mathrm{mmol}) 3$ werden in $10 \mathrm{ml}$ absol. THF gelöst, $0.23 \mathrm{~g}(6 \mathrm{mmol}) \mathrm{NaBH}_{4}$ zugegeben und langsam $0.76 \mathrm{~g}(6 \mathrm{mmol})$ Dimethylsulfat in $2 \mathrm{ml}$ absol. THF zugetropft. Es wird $12 \mathrm{~h}$ bei Raumtemp. gerührt, anschließend mit Ethanol, dann mit $2 \mathrm{~N}-\mathrm{NaOH}$ versetzt und wiederum $1 \mathrm{~h}$ gerührt. Das Lösungsmittel wird abdestilliert, der Rückstand mit Wasser aufgenommen und mit $\mathrm{CHCl}_{3}$ extrahiert, die Lösung mit $\mathrm{Na}_{2} \mathrm{SO}_{4}$ getrocknet und abdestilliert: farblose Kristalle aus Ether von $3-\mathrm{BH}_{3}(0.38 \mathrm{~g}, 90 \%)$.

\section{$\alpha$-Narkotin-Bortrideuteridaddukt $\left(3-B D_{3}\right)$}

$0.83 \mathrm{~g}(2 \mathrm{mmol}) 3$ werden in $8 \mathrm{ml} \mathrm{BF}_{3}$-Etherat gelöst und bei $0^{\circ} \mathrm{zu} 0.16 \mathrm{~g}(4 \mathrm{mmol}) \mathrm{NaBD}_{4}$ in $4 \mathrm{ml} \mathrm{absol}$. THF getropft. Es wird 1 h unter Rückfluß erhitzt; Aufarbeitung s.o. Ausb. $0.85 \mathrm{~g}(99 \%)$, Schmp. $123^{\circ}$ (ab $106^{\circ}$ Zers.), $[\alpha]_{\mathrm{D}}^{20}=+84^{\circ}\left(\mathrm{CHCl}_{3}\right) \cdot \mathrm{C}_{22} \mathrm{H}_{23} \mathrm{NO}_{7} \cdot \mathrm{BD}_{3}$ (430.3) IR (KBr): $1760 \mathrm{~cm}^{-1}$ (CO). MS-FD: $\mathrm{m} / \mathrm{z}=430\left(\mathrm{M}^{+\bullet}\right), 428$. UV (Methanol): $\lambda \max (\log \varepsilon)=226$ (4.28), 289 (sh; 3.52), $310 \mathrm{~nm}$ (3.57).

\section{3,3-Dideutero-1,3-dihydro-4,5-dimethoxy-1-(1,2,3,4-tetrahydro-8-methoxy-2-methyl-6,7-methylendi-} oxy-isochinolyl-1)-isobenzofuran (5) und 5- $B D_{3}$

$0.83 \mathrm{~g}(2 \mathrm{mmol}) 3$ werden in $8 \mathrm{ml} \mathrm{BF}_{3}$-Etherat gelöst und bei $0^{\circ} \mathrm{zu} 0.16 \mathrm{~g}(4 \mathrm{mmol}) \mathrm{LiAlD}_{4}$ in $4 \mathrm{ml} \mathrm{absol}$. THF getropft. Die Lösung wird $2 \mathrm{~h}$ unter Rückfluß erhitzt und nach Abkühlen wie oben aufgearbeitet; farblose Kristalle, die aus Ethanol umkristallisiert werden: 5- $\mathrm{BD}_{3}$. Ausb. $0.80 \mathrm{~g}$ (96\%), Schmp. 146-147,$[\alpha]_{\mathrm{D}}^{20}=+93^{\circ}\left(\mathrm{CHCl}_{3}\right) \cdot \mathrm{C}_{22} \mathrm{H}_{23} \mathrm{D}_{2} \mathrm{NO}_{6} \cdot \mathrm{BD}_{3}(418.3)$ Ber. C $63.1 \mathrm{H} 6.83$, Gef. C62.8 H 6.75. IR (KBr): $1780 \mathrm{~cm}^{-1}$ (CO). MS-FD: $\mathrm{m} / \mathrm{z}=418\left(\mathrm{M}^{+\cdot}\right), 415$. UV (Methanol): $\lambda$ max $(\log \varepsilon)=224$ (4.23), $283 \mathrm{~nm}(3.50)$. - Durch 16-stdg. Erhitzen von 5- $\mathrm{BD}_{3}$ in 20-proz. $\mathrm{KOH}$ erhält man in quantitativer Ausbeute 5, das aus Ethanol umkristallisiert wird: farblose Nadeln, Schmp. $125^{\circ}$, $[\alpha]_{\mathrm{D}}^{20}=-105^{\circ}\left(\mathrm{CHCl}_{3}\right) \cdot \mathrm{C}_{22} \mathrm{H}_{23} \mathrm{D}_{2} \mathrm{NO}_{6}(401.3)$ Ber. C65.8 H 6.29, Gef. C65.7 H 6.06. MS: $\mathrm{m} / \mathrm{z}=220$ (100\%), $205\left(16 \%,{ }^{*} 191.02\right), 181(3 \%)$. MS-FD: $m / z=401\left(M^{+\bullet}\right)$. UV (Methanol): $\lambda \max (\log \varepsilon)=$ 224 (4.38), $281 \mathrm{~nm}(3.52)$.

\section{$\beta$-Hydrastin-Boranaddukt (6- $\left.\mathrm{BH}_{3}\right)$}

$0.77 \mathrm{~g} \beta$-Hydrastin (6) werden wie oben mit $\mathrm{NaBH}_{4} / \mathrm{BF}_{3}$-Etherat unter 1 -stdg. Rühren bei $0^{\circ}$ umgesetzt: $\beta$-Hydrastin-Boranaddukt $\left(6-\mathrm{BH}_{3}\right)$ als farblose Kristalle aus $\mathrm{EtOH}(0.65 \mathrm{~g}, 82 \%)$, Schmp. $167^{\circ},[\alpha]_{\mathrm{D}}^{20}=+278\left(\mathrm{CHCl}_{3}\right) \cdot \mathrm{C}_{21} \mathrm{H}_{21} \mathrm{NO}_{6} \cdot \mathrm{BH}_{3}(397.3)$ Ber. C $63.5 \mathrm{H} 6.09$, Gef. C 63.2 H 5.93. MS

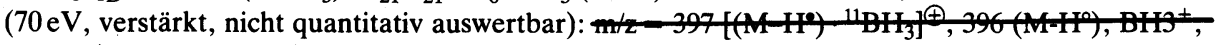

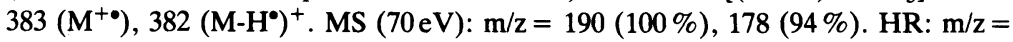
396: $\mathrm{C}_{21} \mathrm{H}_{23}{ }^{11} \mathrm{BNO}_{6}$ Ber. 396.161833 Gef. 396.16211

383: $\mathrm{C}_{21} \mathrm{H}_{21} \mathrm{NO}_{6}$ Ber. 383.136876 Gef. 383.13469

382: $\mathrm{C}_{21} \mathrm{H}_{20} \mathrm{NO}_{6}$ Ber. 382.129052 Gef. 382.13043

190: $\mathrm{C}_{11} \mathrm{H}_{12} \mathrm{NO}_{2}$ Ber. 190.086798 Gef. 190.08696

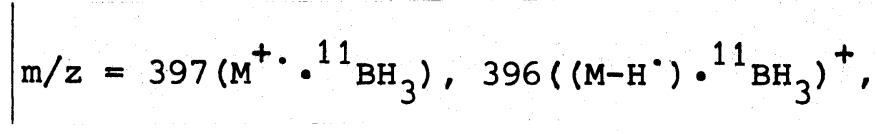


178: $\mathrm{C}_{10} \mathrm{H}_{10} \mathrm{O}_{3}$ Ber. 178.062989 Gef. 178.06378

MS-FD: m/z $397\left(\mathrm{M}^{+\bullet} .{ }^{11} \mathrm{BH}_{3}\right), 395\left(\mathrm{M} \cdot \mathrm{BH}^{+\bullet}\right)$. IR (KBr): $1755(\mathrm{CO}), 2300-2400 \mathrm{~cm}^{-1}\left(\mathrm{BH}_{3}\right)$. UV (Methanol): $\lambda \max (\log \varepsilon)=213$ (4.08), 238 (sh, 3.58), 295 (3.49),315 nm (sh, 3.38).

\section{$\beta$-Anhydrohydrastindiol (7) und 7- $\mathrm{BH}_{3}$}

$0.77 \mathrm{~g} 6$ werden mit $\mathrm{NaBH}_{4} / \mathrm{BF}_{3}$-Etherat wie oben versetzt, aber $1 \mathrm{~h}$ unter Rückfluß erhitzt. Das nach Aufarbeitung (s.o.) erhaltene Öl wird sc (Kieselgel, Essigester) gereinigt. Fraktion Rf 0.8: farblose Kristalle von $\beta$-Anhydrohydrastindiol-Boranaddukt $\left(7-\mathrm{BH}_{3}\right)$, die aus Ethanol umkristallisiert werden $(0.52 \mathrm{~g}, 68 \%)$, Schmp. $128-131^{\circ},[\alpha]_{\mathrm{D}}^{20}=+211^{\circ}\left(\mathrm{CHCl}_{3}\right) \cdot \mathrm{C}_{21} \mathrm{H}_{23} \mathrm{NO}_{5} \cdot \mathrm{BH}_{3}(383.3)$ Ber. C $65.8 \mathrm{H}$ 6.84, Gef. C $65.7 \mathrm{H}$ 6.77. IR (KBr): $2280-2400 \mathrm{~cm}^{-1}\left(\mathrm{BH}_{3}\right) . \mathrm{MS}(70 \mathrm{eV}): \mathrm{m} / \mathrm{z}=382\left[\left(\mathrm{M}-\mathrm{H}^{\circ}\right) \cdot{ }^{11} \mathrm{BH}_{3}^{\oplus}\right.$, $<1 \%$ ], 354 (<1\%), 191 (14\%), 190 (100\%), 179 (6\%). MS-FD: $\mathrm{m} / \mathrm{z}=383\left(\mathrm{M}^{+\bullet} .{ }^{11} \mathrm{BH}_{3}\right), 381$ $\left(\mathrm{M}^{+} \cdot \mathrm{BH}\right)$. UV (Methanol): $\lambda \max (\log \varepsilon)=215$ (4.23), 235 (sh; 3.68), $285 \mathrm{~nm}$ (3.56).

Fraktion Rf 0.3: $\beta$-Anhydrohydrastindiol (7) $(0.10 \mathrm{~g}, 14 \%)$ als farbloses Öl. $-7-\mathrm{BH}_{3} \mathrm{kann}$ durch Erhitzen mit 2N-KOH/Dioxan zu 7 gespalten werden. $-\mathrm{C}_{21} \mathrm{H}_{23} \mathrm{NO}_{5}(369.4),[\alpha]_{\mathbb{D}}^{20}=+51^{\circ}\left(\mathrm{CHCl}_{3}\right)$. UV (Methanol): $\lambda \max (\log \varepsilon)=217$ (4.22), $286 \mathrm{~nm}(3.50)$.

\section{2-(3,4-Dimethoxyphenyl)-2a,3,4,5-tetrahydro-3-methyl-7,8-methylendioxy-2H-furo [2,3,4-i,k] iso- chinolin (9)}

$4.17 \mathrm{~g}(10 \mathrm{mmol}) 8,9.50 \mathrm{~g}(52 \mathrm{mmol})$ trockenes Benzophenon und $2.90 \mathrm{~g}(26 \mathrm{mmol}) \mathrm{K}$-tert. butylat werden in $50 \mathrm{ml}$ absol. Benzol unter $\mathrm{N}_{2} 6 \mathrm{~h}$ zum Sieden erhitzt. Nach Abkühlen werden die Produkte mit verd. $\mathrm{HCl}$ extrahiert. Die wäßrige Phase wird mit Ether benzolfrei gewaschen und unter Eiskühlung mit überschüssigem $\mathrm{NH}_{3}$ versetzt. Die Mischung wird mit $\mathrm{CHCl}_{3}$ extrahiert, die org. Phase mit $\mathrm{Na}_{2} \mathrm{SO}_{4}$ getrocknet und abdestilliert. Das Öl wird in wenig Ethanol heiß gelöst. Nach dem Abkühlen fällt Ausgangsmaterial $8(2.50 \mathrm{~g}, 60 \%)$ aus. Der Überstand wird eingeengt, die Produkte werden sc (Kieselgel; Essigester) getrennt: Fraktion Rf 0.7: Die Kristalle sind mit $\alpha$-Narkotin (3) identisch (Ausb. $0.53 \mathrm{~g}, 13 \%$ ).

Fraktion Rf 0.6: Kristalle aus Ether, die aus Ethanol umkristallisiert werden: 9

Ausb. 0.40g (11\%), Schmp. $167^{\circ},[\alpha]_{\mathrm{D}}^{20}=+45^{\circ}\left(\mathrm{CHCl}_{3}\right) \cdot \mathrm{C}_{20} \mathrm{H}_{21} \mathrm{NO}_{5}(355.4) \mathrm{MS}: \mathrm{m} / \mathrm{z}=355\left(\mathrm{M}^{+\bullet}\right.$, $48 \%), 218\left(46 \%,{ }^{*} 133.87\right), 189(100 \%), 159(43 \%)$.

MS-HR: $\mathrm{C}_{20} \mathrm{H}_{21} \mathrm{NO}_{5}$ : Ber. 355.14196 Gef. 355.14150

$\mathrm{C}_{12} \mathrm{H}_{12} \mathrm{NO}_{3}$ : Ber. 218.08171 Gef. 218.08191

$\mathrm{C}_{11} \mathrm{H}_{11} \mathrm{NO}_{2}$ : Ber. 189.07897 Gef. 189.07886

$\mathrm{C}_{10} \mathrm{H}_{9} \mathrm{NO}$ : Ber. 159.06841 Gef. 159.06832

${ }^{1} \mathrm{H}-\mathrm{NMR}(90 \mathrm{MHz}): \delta(\mathrm{ppm})=2.12\left(\mathrm{~s} ; 3 \mathrm{H},-\mathrm{NCH}_{3}\right), 2.38-3.13\left(\mathrm{~m} ; 4 \mathrm{H},-\mathrm{CH}_{2}-\right), 3.69\left(\mathrm{~d}\right.$ von t; $\mathrm{J}_{\mathrm{d}}=$ $\left.10.1 \mathrm{~Hz}, \mathrm{~J}_{\mathrm{t}}=1.5 \mathrm{~Hz}, 1 \mathrm{H},-\mathrm{N}-\mathrm{CH}-\right), 3.90\left(\mathrm{~s} ; 3 \mathrm{H},-\mathrm{OCH}_{3}\right), 3.91\left(\mathrm{~s} ; 3 \mathrm{H},-\mathrm{OCH}_{3}\right), 5.32(\mathrm{~d} ; \mathrm{J}=10.1 \mathrm{~Hz}$, $1 \mathrm{H},-\mathrm{O}-\mathrm{CH}-), 5.86,5.90\left(\mathrm{AB} ; \mathrm{J}=1.3 \mathrm{~Hz}, 2 \mathrm{H},-\mathrm{O}-\mathrm{CH}_{2}-\mathrm{O}-\right), 6.27$ (s; $1 \mathrm{H}$, Aromaten-H), 6.90 (d; J = $8.6 \mathrm{~Hz}, 1 \mathrm{H}$, Aromaten-H) 7.05-7.27 (m; 3H, Aromaten-H). UV (Methanol): $\lambda$ max $(\log \varepsilon)=217$ (4.31), $280 \mathrm{~nm}(3.51)$.

\section{2-(3,4-Dimethoxyphenyl)-3-dimethylamino-6,7-methylendioxy-4-vinyl-dihydrobenzofuran (11)}

$0.70 \mathrm{~g}(2 \mathrm{mmol}) 9$ werden $20 \mathrm{~h}$ in $25 \mathrm{ml} \mathrm{CH}_{3} \mathrm{I}$ unter Rückfluß erhitzt, die Kristalle werden getrocknet: 9- $\mathrm{CH}_{3} \mathrm{I}$ (Ausb. $0.81 \mathrm{~g}, 82 \%$ ) Schmp. $243^{\circ} .0 .70 \mathrm{~g}$ 9- $\mathrm{CH}_{3} \mathrm{I}$ werden in $50 \mathrm{ml} 20$-proz. methanol. KOH $5 \mathrm{~h}$ unter Rückfluß erhitzt. Das nach Ausschütteln mit $\mathrm{CHCl}_{3}$, Trocknen mit $\mathrm{Na}_{2} \mathrm{SO}_{4}$ und Abdestillieren des Solvens erhaltene Öl wird sc (Kieselgel; $\mathrm{CHCl}_{3}$ ) gereinigt: 10 als farbloses Öl. Ausb. $0.50 \mathrm{~g}$ $(68 \%),[\alpha]_{\mathrm{D}}^{20}=-32^{\circ}\left(\mathrm{CHCl}_{3}\right) \cdot \mathrm{C}_{21} \mathrm{H}_{23} \mathrm{NO}_{5}(369.3) \mathrm{MS}: \mathrm{m} / \mathrm{z}=369\left(\mathrm{M}^{+}, 65 \%\right), 354(3 \%), 340(2 \%)$, $326\left(30 \%,{ }^{*} 288.01\right), 325\left(100 \%,{ }^{*} 286.25\right), 309(4 \%), 295(13 \%, * 267.77), 267(12 \%, * 241.66), 217$ (6\%), $202(12 \%), 194(14 \%), 184.5\left(\mathrm{M}^{2+}, 6 \%\right), 165(15 \%)$. 
${ }^{1} \mathrm{H}-\mathrm{NMR}(60 \mathrm{MHz}): \delta(\mathrm{ppm})=2.30\left(\mathrm{~s} ; 6 \mathrm{H},-\mathrm{N}\left(\mathrm{CH}_{3}\right)_{2}\right), 3.85\left(\mathrm{~s} ; 6 \mathrm{H},-\mathrm{OCH}_{3}\right), 4.43(\mathrm{~d} ; \mathrm{J}=2.5 \mathrm{~Hz}, 1 \mathrm{H}$, $-\mathrm{CH}-\mathrm{N}), 5.20\left(\mathrm{AB} ; \mathrm{J}=1 \mathrm{~Hz}, \mathrm{~J}^{\prime}=11 \mathrm{~Hz}, 1 \mathrm{H}\right.$, Vinyl-H), $5.65\left(\mathrm{AB} ; \mathrm{J}=1 \mathrm{~Hz}, \mathrm{~J}^{\prime}=17 \mathrm{~Hz}, 1 \mathrm{H}\right.$, Vinyl-H), $5.73(\mathrm{~d} ; \mathrm{J}=2.5 \mathrm{~Hz}, 1 \mathrm{H},-\mathrm{CH}-\mathrm{O}-), 6.00\left(\mathrm{~s} ; 2 \mathrm{H},-\mathrm{OCH}_{2} \mathrm{O}-\right), 6.73-7.08(\mathrm{~m} ; 5 \mathrm{H}$, Aromaten- $\mathrm{H}, 1 \mathrm{H}$, Vinyl-H). UV Methanol: $\lambda \max (\log \varepsilon)=231(3.90), 279 \mathrm{~nm}(3.58)$.

\section{Literatur}

1 W. Wiegrebe, S. Prior und K.K. Mayer, Arch. Pharm. (Weinheim) 315, 262 (1982).

2 H.C. Brown, D.B. Bigley, S.K. Arora und N.M. Yoon, J. Am. Chem. Soc. 92, 7161 (1970).

3 S. Yamada und S. Ikegami, Chem. Pharm. Bull. 14, 1382 (1966).

4 G.R. Pettit und T.R. Kasturi, J. Org. Chem. 26, 4557 (1961).

5 M. Shamma und V.St. Georgiev, Tetrahedron Lett. 1974, 2339.

6 S. Safe und R.Y. Moir, Can. J. Chem. 42, 160 (1964); M. Shamma und V.St. Georgiev, Tetrahedron 32, 211 (1976).

7 K.W. Bentley und A.W. Murray, J. Chem. Soc. 1963, 2487.

8 M.H. Abu Zarga, G.A. Miana und M. Shamma, Tetrahedron Lett. 1981, 541.

9 S. Chattopadhyay und M. Shamma, Heterocycles 19, 697 (1982).

10 T.J. Batterham, NMR Spectra of Simple Heterocycles, S. 375-377, J. Wiley and Sons, New York 1973.

[Ph 644] 